\title{
Indications of microscopic solvability from counting arguments
}

\author{
Wim van Saarloos and John D. Weeks \\ AT\&T Bell Laboratories, Murray Hill, New Jersey 07974-2070 \\ Martin Grant \\ AT\&T Bell Laboratories, Murray Hill, New Jersey 07974-2070 \\ and Department of Physics, McGill University, Montreal, Québec, Canada H3A $2 T 8^{*}$
}

(Received 6 July 1987)

\begin{abstract}
Recent analytical results for the shape of viscous fingers in a Hele-Shaw cell have established that the steady-state equations with nonzero surface tension are overdetermined and hence do not admit finger solutions with arbitrary finger widths. We develop a simple counting argument for the asymptotic region (the tails of the finger), based on the symmetry of the system for zero surface tension, which indicates in a simple way how this overcompleteness arises. Similarities and differences with counting arguments for breather solutions in soliton theory and for dendritic needle solutions are also briefly discussed.
\end{abstract}

In the last few years, substantial progress has been made in resolving the long-standing issue of the "selection" of the finger width in Hele-Shaw cells. In the classical work of Saffman and Taylor, ${ }^{1,2}$ the effects of the surface tension $\sigma$ at the interface were neglected, and a continuous family of steady-state finger solutions was found. A perturbation analysis ${ }^{3}$ for small values of the capillary constant indicated that this continuous family of solutions continued to exist for nonzero $\sigma$. Experiments, however, found a unique finger width for fixed values of the experimental parameters. ${ }^{1,2}$

Recent analysis by several groups ${ }^{4-6}$ has shown that this discrepancy arises because the surface tension acts as a singular perturbation; with $\sigma$ nonzero there can exist only a discrete set of steady-state solutions. These findings are thus in agreement with the "microscopic solvability" scenario, ${ }^{7}$ which had first been suggested on the basis of studies of simple local models. ${ }^{8,9}$

The most useful qualitative test that indicates for a particular problem whether or not singular perturbations are important has turned out to be a "counting argument." For example, in the geometrical model ${ }^{9}(\mathrm{GM})$, the fact that there are in general no steady-state finger solutions for arbitrarily small surface tension can be seen quite easily from a simple argument based on counting the number of convergent and divergent modes near the fixed point describing the properties of the finger solutions in the tails. ${ }^{9}$ This counting argument not only allowed a simple intuitive understanding of how seemingly small terms involving the surface tension could have large and singular effects, but also was a crucial step in the rigorous mathematical analysis of the GM.

A second example of the power of a counting argument in cases where singular perturbations can arise is provided by recent results for breather solutions in the $\phi^{4}$ model, i.e., solutions that are localized in space and periodic in time. A simple counting argument ${ }^{10,11}$ involving the number of conditions that the Fourier modes $a_{n}(x)$, with $0 \leq n \leq \infty$, must obey in the asymptotic re- gions $x \rightarrow \pm \infty$ shows that in general no nontrivial breathers should be found, even though numerical evidence seemed to indicate the existence of breathers. Recently the nonexistence of breathers was confirmed by a rigorous mathematical analysis of the $\phi^{4}$ equation for small-amplitude breathers. ${ }^{12}$

It is natural to ask whether a similar counting argument in the tails of viscous fingers using the properties of the full nonlocal equations can give an indication of the fact that here also the surface tension is a singular perturbation. This is the issue we wish to address in this paper. Our analysis also highlights some of the differences between the tail region of needle crystals and those of viscous fingers and has some bearing on an aspect of the solvability method that has not received much attention. However, we are not able to clarify the relation between the counting argument in the tails and the general structure of the Mullins-Sekerka instability, which in most approaches is fundamentally connected with solvability.

At first sight, it may seem surprising that the analytical structure of the local models and the viscous fingering problem are so close, since the pressure field governing the fluid flow in viscous fingering obeys the Laplace equation ${ }^{1,2}$ and is therefore long ranged. As a result, the dynamics of the interface at one point depends on the shape everywhere else, whereas in the local models ${ }^{8,9}$ the dynamics only depends on local quantities like the curvature and its derivatives. Mathematically, the connection is demonstrated most clearly in the recent work by Bensimon et al., ${ }^{13}$ who show that when the dynamical equation for small short-wavelength perturbations obeying the Laplace equation is analytically continued into the complex plane, the resulting equation is reminiscent of the one defining the GM. Physically, the reason why viscous fingering is in a sense rather "local" is that when the compressibility of the fluid is taken into account, the appropriate diffusion length is many orders of magnitude larger than the cell width. ${ }^{14}$ Thus, the walls are always 
"close" and as a result the most important asymptotic properties of the finger shape can be determined on the basis of an analysis in the tail region only.

To see this, let the Hele-Shaw cell occupy the region $-1 \leq x \leq 1$ of the $x-z$ plane. In dimensionless coordinates, the shape of a steady-state viscous finger moving with velocity $V$ in the $z$ direction is then determined by the Laplace equation for the pressure field $p$ and the appropriate boundary conditions, 1,2

$$
\begin{aligned}
& \nabla^{2} p=0, \\
& p_{i}=-\sigma \kappa, \\
& -\hat{\mathbf{n}} \cdot \nabla p_{i}=\cos \theta,
\end{aligned}
$$

where $\sigma$ is the dimensionless surface tension, $\hat{\mathbf{n}}$ the normal to the interface, $\kappa$ the interface curvature, $\theta$ the angle $\hat{\mathbf{n}}$ makes with the $z$ axis, and $p_{i}$ the pressure at the interface. Anticipating exponential convergence of the finger width to its limiting value $\lambda$ as $z \rightarrow-\infty$, we write for the right interface in the moving frame with the tip at $z=0$

$$
x=\lambda+a_{1} e^{t z}+\cdots .
$$

With this ansatz, the pressure field in the gap between the cell walls and the interface will also have an $e^{t z}$ dependence, and the solution that satisfies the Laplace equation and the boundary condition $\partial p /\left.\partial x\right|_{x=1}=0$ at the right cell wall is

$$
p=-A e^{t z} \cos [t(1-x)] \text {. }
$$

Upon linearization of Eqs. (1b) and (1c), we then get $A=a_{1} / \sin [t(1-\lambda)]$ and

$$
t^{-2} \cot [t(1-\lambda)]=\sigma \text {. }
$$

This result was first derived by McLean and Saffman. ${ }^{3}$ The derivation given here follows that of Kessler et al. ${ }^{15}$ Equation (4) explicitly shows that the exponent $t$ is completely determined by an analysis of the "outer expansion" for the "tail" region of the viscous fingers. Moreover, by extending this analysis to higher order with the assumption

$$
x=\lambda+a_{1} e^{t z}+a_{2} e^{2 t z}+a_{3} e^{3 t z}+\cdots,
$$

the coefficients $a_{2}, a_{3}, \ldots$, etc., can be determined ${ }^{3,15}$ uniquely in terms of $a_{1}$; thus, to each "mode" $e^{t z}$ there corresponds one unique solution of the outer expansion. (The fact that $a_{1}$ is undetermined has no significance, since it corresponds to the translational freedom of the finger in the $z$ direction.)

Note that, since the cotangent is periodic, Eq. (4) has an infinite number of solutions with $t$ positive as well as negative - in fact, in the limit $\sigma \rightarrow 0$, the solutions become symmetric, $t= \pm\left(\frac{1}{2} \pi+n \pi\right) /(1-\lambda), n=0,1,2, \ldots$ This latter symmetry expresses the fact that Eqs. (1) for $\sigma=0$ are invariant under a reversal of the flow direction, so that the Saffman-Taylor solutions describe retreating as well as advancing fingers. To see this, note that if $p(x, z)$ is the pressure field associated with an advancing Saffman-Taylor $\sigma=0$ finger solution, then the pressure field $\widetilde{p}=-p$ solves the equations $\nabla^{2} \widetilde{p}=0, \widetilde{p}_{i}=0$, $\hat{\mathbf{n}} \cdot \nabla \widetilde{p}_{i}=\cos \theta$, which describe a retreating SaffmanTaylor finger of exactly the same shape with velocity $\widetilde{V}=-V$. [In dimensional form, the velocity $V$ appears on the right side of Eq. (1c).] It is convenient also to set $z \rightarrow-z$, so that both fingers move in the positive $z$ direction, with the tails of the original advancing finger found as $z \rightarrow-\infty$ and those of the "retreating" finger found as $z \rightarrow+\infty$. Clearly only positive $t$ modes in (2) are acceptable for the advancing finger as $z \rightarrow-\infty$, while negative exponents $\tilde{t}<0$ are required for the "retreating" finger as $z \rightarrow \infty$. The invariance of the Saffman-Taylor equations under a change in flow direction than implies the mode symmetry $\tilde{t}=-t$. (For the same reason, rising bubbles are symmetric ${ }^{16}$ for $\sigma=0$.)

Since there is an infinite number of modes $t_{n}$ satisfying Eq. (4), the proper counting is not completely obvious. However, there is a pairing of convergent and divergent modes in the limit $\sigma \rightarrow 0$, and the only natural truncation that preserves this symmetry is to take the same number of convergent and divergent modes even with $\sigma$ nonzero but small.

Once this is agreed to, however, it is clear that the finger problem is in general overdetermined. If there are $N$ convergent and $N$ divergent modes at each end of the finger, then there are $N$ conditions at, say, the righthand side of the finger $(x>0)$ that must be satisfied so that no divergent (for $z \rightarrow-\infty$ ) modes are present. However, there effectively are only $N-1$ free parameters available from the coefficients of the $N$ convergent modes on the left-hand side $(x<0)$ which could be adjusted to bring this about; one degree of freedom describes only a trivial overall translation of the finger in the $z$ direction, as is easily seen from (2), and this cannot help eliminate any of the divergent modes at $x>0$. Since we are therefore lacking one parameter, we in general do not expect to find a smooth finger solution satisfying the physical boundary conditions at the sidewalls for arbitrary finger width $\lambda$.

Since the problem appears to be overdetermined by one condition, this picture is close to that emerging from the local models. ${ }^{8,9}$ Moreover, it is consistent with the finding ${ }^{4-6,13}$ that there are solutions for particular values of $\lambda$, as well as with the idea underlying the numerical approaches ${ }^{17,7}$ that one also obtains a well-posed problem with a unique solution for arbitrary $\lambda$ upon relaxing one condition (usually, one relaxes the condition $d z / d x=0$ at $x=0$ ). However, since the above counting argument does not distinguish between $\sigma=0$ and $\sigma \neq 0$, it also implies that one would never in general expect the existence of a continuous family of solutions. Thus, in this picture the continuous family of Saffman-Taylor solutions ${ }^{2}$ must arise because of some accident, probably caused by the additional symmetry present at $\sigma=0$. In this case the "modes" can couple since a third-order term of a mode with $t=\pi /[2(1-\lambda)]$ is of the same order as a linear mode with $t=3 \pi /[2(1-\lambda)]$. If this idea is correct, the viscous fingering problem resembles to some extent the breather problem ${ }^{12}$ since in that case a counting argument would also lead one to believe that the existence of breather solutions is virtually impossible; 
nevertheless, the sine-Gordon equation does admit breather solutions. ${ }^{18}$ This is discussed in more detail in the Appendix.

It is interesting to compare the above counting argument for viscous fingers to what has been found for dendritic growth. To the best of our knowledge, there has been no direct analysis of modes in the tails of needle crystals analogous to (4). Indeed, dendritic needles do not approach the asymptotic Ivantsov shape exponentially fast; rather, there are terms involving powers of $x^{-1}$ and terms with fractional exponents in two dimensions in the absence of interface kinetics. ${ }^{19}$ Furthermore, the buildup of latent heat released at earlier times in the boundary layer in front of the growing interface gives the needle crystal problem an additional temporal nonlocality ${ }^{7 b, 14}$ that makes a complete analysis more difficult to carry out.

However, in contrast to the Saffman-Taylor finger satisfying the Laplace equation, the equations for the Ivantsov needles are not invariant to a reversal of the growth direction. That is, the steady-state diffusion equation $V \partial T / \partial z+D \nabla^{2} T=0$ for the temperature field changes under the transformation $V \rightarrow-V, T \rightarrow-T$, so that growing needles differ from melting ones. In fact, there are no steady-state melting-needle solutions at all. As a result, there is no symmetry that dictates a straightforward counting argument such as the one we made for the Saffman-Taylor finger. Indeed, a physical interpretation ${ }^{20}$ of the strong asymmetry in the tail region suggests that most modes are convergent on approaching the tail region of the needle crystal.

Two of us $^{2}$ argued that this behavior could give rise to a continuous family of steady-state needle solutions. On the other hand, needle crystals may be different from viscous fingers in that behavior in the tail region could be of little physical relevance for the possible existence of steady-state solutions, particularly at small Péclet numbers. ${ }^{20}$ Until there has been a proper matching of the "outer expansion" results ${ }^{20}$ appropriate for the tails of needle crystal to the "inner region" near the tip, ${ }^{21}$ this point remains unresolved.

It is unfortunate that there does not seem to be, at present, a more direct way to establish whether the steady-state solutions of interface problems governed by integral equations are in fact overdetermined for nonzero surface tension, because the uniqueness of solutions after dropping a sufficient number of boundary conditions is an essential part of the "asymptotic beyond all orders" method of Kruskal and Segur as applied to the geometrical model ${ }^{22}$ and the breather problem of $\phi^{4}$ theory. ${ }^{12}$

The first step in this approach ${ }^{12,22}$ is to drop a boundary condition in order to obtain a well-posed problem with a unique solution, which reduces to the smooth steady-state shape of a finger ${ }^{22}$ or the breather, ${ }^{12}$ if such solutions exist. To convince oneself of the uniqueness of the modified problem, a type of counting argument is performed for the asymptotic behavior in the tails of the solutions.

In the second step of the analysis, the (a)symmetry of the solution is investigated at a special point at which the asymptotic series converges trivially (the tip for nee- dles, $x=0$ for breathers). The exponentially small but singular term that shows the asymmetry of solutions at this special point (and hence which shows that no globally valid symmetric solutions exist) can be obtained from an analysis near a singularity in the complex plane. Since we know that the solution is unique, we can conclude that physically valid solutions of the original problem exist only in those special cases where the asymmetry happens to vanish.

Most workers ${ }^{15,23-27}$ have proceeded in much the same way for the needle crystal problem, and implicitly assumed that solutions are uniquely defined after dropping a boundary condition, usually the requirement that $d z / d x$ is zero at the tip. As stressed before, however, we do not have a counting argument to help us decide whether or not this is likely to be the case. The uniqueness assumption is important since the modified problem obtained by relaxing a boundary condition might on physical grounds generally be expected to have a solution. Indeed, Hong and Langer ${ }^{28}$ have already suggested that the generalized problem for the Hele-Shaw cell could describe the fingers with a bubble at the tip observed by Couder et al. ${ }^{29,30}$ and by Maxworthy, ${ }^{31}$ and one could similarly envision some grain-boundary-like defect to permit a finite cusp at the tip during crystal growth. Thus the fact that the analytic methods generally find the prefactor ( $\Gamma$ in the analysis of Kruskal and Segur $^{22}$ of the GM) of the singular term nonzero is not necessarily surprising, since relaxing the boundary condition has introduced additional freedom. Unless uniqueness can be established, one cannot rule out the existence of other (smooth) solutions.

One should also keep in mind that if the problem is overdetermined by more than one condition (as happens, e.g., for the breathers - see the Appendix), the solution that numerically appears to be found upon relaxing one boundary condition will probably not be a true solution after all. However, most approaches that have been introduced so far for interfacial problems are consistent with the idea that these are generally overdetermined by just one condition.

Thus, in contrast to the situation for viscous fingering, we believe the uniqueness of solutions for dendritic growth remains an important open question. A resolution of this issue is also needed to assess the reliability of the numerical methods, ${ }^{17,23,27}$ which use Newton's method to obtain the interface shape after dropping a boundary condition at the tip. If multiple solutions could exist, then only the one with the largest numerical basin of attraction would usually be found by this procedure. If, on the other hand, these problems are overdetermined by more than one condition, the present numerical methods would also fail.

Clearly, "solvability" is, a priori, likely to apply in cases similar to viscous fingering and the breather problem, where a counting argument makes the existence of a continuous family of solutions appear extremely improbable. Intuitively, we expect this situation to occur generally in situations where, as a result of a special symmetry (like the aforementioned symmetry of the modes for $\sigma=0$ in viscous fingering), there are as many 
"convergent" as "divergent" modes in the tails. The Ivantsov needle solutions certainly have an additional special feature because of the absence of an intrinsic length scale, ${ }^{8}$ but, to our knowledge, this simplification is not related to any symmetric pairing or coupling of convergent and divergent modes in the tails. The development of a generalized counting argument appropriate for partial differential equations or integro-differential equations $^{32}$ is needed to resolve this subtle, but important, issue.

The counting argument developed here applies specifically to the asymptotic (tail) region of finger solutions. In the recent approach of Bensimon et al. ${ }^{13}$ an effective mode analysis is done in the tip region; in their formulation, solvability arises from the general structure of the Mullins-Sekerka instability. ${ }^{13}$ The relation between these two pictures is not completely clear to us; it is possible that even if the counting argument in the tails predicts a family of solutions, solvability could still be dynamically relevant.

A related question that we feel needs to be studied in more detail in systems for which solvability is known to hold is what determines the time scale on which the effect of the absence of a true steady-state solution make itself felt. ${ }^{14}$ Even for viscous fingering, ${ }^{4-6}$ where there seems to be no doubt of the relevance of solvability selection for most experiments, this question has become of interest because of recent computer simulations ${ }^{33}$ that give fingers with widths less than $\frac{1}{2}$. Moreover, approximate "breathers" in $\phi^{4}$ theory decay only very slowly ${ }^{12}$ [energy $\sim(\ln t)^{-1}$ ] and are found in numerical simulations. $^{34}$ They could therefore still be physically relevant in certain circumstances. ${ }^{12}$

We are grateful to $\mathbf{H}$. Segur for many illuminating discussions. One of us (M.G.) acknowledges support of the National Science Foundation (NSF) through Grant No. DMR-83-12958.

\section{APPENDIX: COUNTING ARGUMENTS FOR BREATHER SOLUTIONS} form

Consider nonlinear partial differential equations of the

$$
\frac{\partial^{2} u}{\partial t^{2}}-\frac{\partial^{2} u}{\partial x^{2}}=g(u), \quad g(0)=0, g^{\prime}(0)>0 .
$$

With the specific choice $g(u)=\sin u$, this equation becomes the sine-Gordon equation. This equation admits so-called breather solutions: ${ }^{18}$ Solutions which are localized in space (i.e., $u$ decaying exponentially for $x \rightarrow \pm \infty$ ) and periodic in time. For other functions $g(u)$, no exact breather solutions are known, but apparently valid solutions are found in a small-amplitude expansion. However, a type of counting argument ${ }^{10,11}$ indicates that the breather problem is in fact overdetermined, so that no periodic breathers should in general exist. This was proven recently by Segur and Kruskal ${ }^{12}$ for smallamplitude breathers in $\phi^{4}$ theory $\left[g(u)=-2 u+3 u^{2}\right.$ $\left.-u^{3}\right]$. Here, we reformulate the counting argument in a way more closely related to the one discussed above for viscous fingering. ${ }^{35}$

If a real periodic breather solution $u(x, t)$, with frequency $\omega$ exists, it can be expanded in a Fourier series, $u(x, t)=\sum_{n} a_{n}(x) \exp ($ in $\omega t)$, with $a_{-n}(x)=a_{n}^{*}(x)$. The functions $a_{n}(x)$ then satisfy the differential equations ${ }^{12}$

$\left(\partial_{x}^{2}+n^{2} \omega^{2}-2\right) a_{n}=-3 \sum_{m} a_{m} a_{n-m}+\sum_{k, m} a_{k} a_{m} a_{n-k-m}$.

For localized breathers, the $a_{n} \rightarrow 0$ for $x \rightarrow \pm \infty$. Since it is known that breathers with $\omega>\sqrt{2}$ cannot exist, ${ }^{36}$ we consider for concreteness the regime $1<\omega<\sqrt{2}$ (the argument for smaller frequencies is essentially the same). In that case, the asymptotic behavior of the $a_{n}$, according to the linear part of (A2), is of the form for $x \rightarrow-\infty$,

$a_{0}=A_{+}^{0} e^{x \sqrt{2}}+A_{-}^{0} e^{-x \sqrt{2}}$,

$a_{1}=A_{+}^{1} e^{x\left(2-\omega^{2}\right)^{1 / 2}}+A_{-}^{1} e^{-x\left(2-\omega^{2}\right)^{1 / 2}}$,

$a_{n}=A_{+}^{n} e^{i x\left(n^{2} \omega^{2}-2\right)^{1 / 2}}+A_{-}^{n} e^{-i x\left(n^{2} \omega^{2}-2\right)^{1 / 2}}, \quad n \geq 2$

and for $x \rightarrow \infty$,

$a_{0}=B_{+}^{0} e^{x \sqrt{2}}+B_{-}^{0} e^{-x \sqrt{2}}$,

$a_{1}=B_{+}^{1} e^{x\left(2-\omega^{2}\right)^{1 / 2}}+B_{-}^{1} e^{-x\left(2-\omega^{2}\right)^{1 / 2}}$,

$a_{n}=B_{+}^{n} e^{i x\left(n^{2} \omega^{2}-2\right)^{1 / 2}}+B_{-}^{n} e^{-i x\left(n^{2} \omega^{2}-2\right)^{1 / 2}}, \quad n \geq 2$

Since the solution of a second-order differential equation contains two integration constants, we can view the coefficients $A_{ \pm}^{n}$ as the parameters that specify the solution. The coefficients $B_{ \pm}^{n}$ then are determined by integrating the equations up from large negative $x$ to large positive $x$.

If we look for localized breather solutions, the coefficients $A_{-}^{0}$ and $A_{-}^{1}$ have to be zero. Moreover, (A3a) shows that all $A_{+}^{n}$ and $A_{-}^{n}$ for $n \geq 2$ have to be zero, and hence the solution that is localized on the left $(x \rightarrow-\infty)$ can be specified completely by the coefficients $A_{+}^{0}$ and $A_{+}^{1}$. [These correspond to three parameters, since $A_{+}^{0}$ is real $\left(a_{0}=a_{0}^{*}\right)$ while $A_{+}^{1}$ can be complex; however, a change in amplitude and phase in $A_{+}^{1}$ only corresponds to a translation in space and time of the solution.]

When the equations are integrated up to large $x$, there is no $a$ priori reason to expect the coefficients $B^{n}$ to vanish. However, for the solution to be localized on the right $(x \rightarrow \infty)$, the coefficients $B_{+}^{0}, B_{+}^{1}$ and all $B_{ \pm}^{n}$, $n \geq 2$, have to be zero. This requirement therefore leads to an infinite number of conditions, whereas the solution which is localized on the left has only a few free parameters $\left(\boldsymbol{A}_{+}^{0}, \boldsymbol{A}_{+}^{1}\right.$, and $\left.\omega\right)$. Clearly, this counting argument shows that the breather problem is "infinitely overdetermined" and therefore that the existence of breathers for arbitrary function $g(u)$ is extremely unlikely; the infinite number of conditions will at least highly constrain the functions $g(u)$ that can give rise to breathers.

As discussed in the main text, in the "asymptotics beyond all orders" approach it is important to drop some boundary conditions so that a well-posed problem is obtained with a unique solution. ${ }^{12,22}$ Segur and 
Kruskal $^{12}$ do this by studying the left-localized solutions obtained by choosing $A_{+}^{1}$ such that a power series expansion in $\epsilon=\left(2-\omega^{2}\right)^{1 / 2}$ gives a symmetric solution $\left[a_{n}(x)=a_{n}(-x)\right]$, and by specifying one additional condition. ${ }^{37}$ They then proceed to show that the derivatives $\left.\partial_{x} a_{n}\right|_{x=0}$ of this uniquely defined solution contain exponentially small terms in $\epsilon$, which proves the absence of genuine breather solutions. ${ }^{12}$
Finally, we point out that, while the existence of a continuous family of Saffman-Taylor finger solutions is difficult to understand from the point of view of the counting argument, their existence may be related to an additional symmetry. Breather solutions do indeed arise in the sine-Gordon equation because this equation is integrable and allows an infinite number of conservation laws, ${ }^{18,38}$ besides conservation of momentum and energy.

*Present and permanent address.

${ }^{1}$ See for a review, e.g., P. G. Saffman, J. Fluid Mech. 173, 73 (1986); D. Bensimon, L. P. Kadanoff, S. Liang, B. I. Shraiman, and C. Tang, Rev. Mod. Phys. 58, 977 (1986).

${ }^{2}$ P. G. Saffman and G. I. Taylor, Proc. R. Soc. (London) Ser. A 245, 312 (1958).

${ }^{3}$ J. W. McLean and P. G. Saffman, J. Fluid Mech. 102, 455 (1981).

${ }^{4}$ B. I. Shraiman, Phys. Rev. Lett. 56, 2028 (1986).

${ }^{5}$ D. C. Hong and J. S. Langer, Phys. Rev. Lett. 56, 2032 (1986).

${ }^{6}$ R. Combescot, T. Dombre, V. Hakim, Y. Pomeau, and A. Pumir, Phys. Rev. Lett. 56, 2036 (1986).

${ }^{7}$ See for reviews, e.g., (a) D. Kessler, J. Koplik, and H. Levine, Adv. Phys. (to be published) (b) J. S. Langer, Les Houches Lecture Notes (unpublished).

${ }^{8}$ E. Ben-Jacob, N. Goldenfeld, J. S. Langer, and G. Schön, Phys. Rev. Lett. 51, 1930 (1983); Phys. Rev. A 29, 330 (1984); E. Ben-Jacob, N. Goldenfeld, G. Kotliar, and J. S. Langer, Phys. Rev. Lett. 53, 2110 (1984).

${ }^{9}$ R. Brower, D. A. Kessler, J. Koplik, and H. Levine, Phys. Rev. Lett. 51, 1111 (1983); Phys. Rev. A 29, 1335 (1984); D. A. Kessler, J. Koplik, and H. Levine, ibid. 30, 2820 (1984); 30, 3161 (1984); 31, 1713 (1985).

${ }^{10}$ V. M. Elonskii, N. E. Kulagin, N. S. Novozhilova, and V. P. Silin, Teor. Mat. Fiz. 60, 395 (1984).

${ }^{11}$ A. Weinstein, Commun. Math. Phys. 99, 385 (1985); 107, 177 (1986); and unpublished lecture notes of the Defense Advanced Research Projects Agency (DARPA) research group conference, Berkeley, 1986.

${ }^{12}$ H. Segur and M. D. Kruskal, Phys. Rev. Lett. 58, 747 (1987); 58, 1158(E) (1987).

${ }^{13}$ D. Bensimon, P. Pelcé, and B. I. Shraiman, J. Phys. (Paris) (to be published).

${ }^{14}$ This is discussed in more detail by J. D. Weeks and W. van Saarloos, Phys. Rev. A 35, 3001 (1987). See also W. van Saarloos and J. D. Weeks, Phys. Rev. Lett. 55, 1685 (1985).

${ }^{15}$ D. A. Kessler, J. Koplik, and H. Levine, Phys. Rev. A 34, 4980 (1986).

${ }^{16}$ G. I. Taylor and P. G. Saffman, Q. J. Mech. Appl. Math. 12, 265 (1959).

${ }^{17}$ J. M. Vanden-Broeck, Phys. Fluids 26, 2033 (1983).

${ }^{18}$ See, e.g., M. J. Ablowitz and H. Segur, Solitons and the Inverse Scattering Transform (SIAM, Philadelphia, 1981).

${ }^{19}$ W. van Saarloos, J. D. Weeks, and B. G. Kotliar, Phys. Rev. A 35, 2288 (1987).

${ }^{20}$ W. van Saarloos and J. D. Weeks, Phys. Rev. A 35, 2357 (1987).

${ }^{21}$ P. Pelcé and Y. Pomeau, Stud. Appl. Math. 74, 245 (1986).

${ }^{22}$ M. D. Kruskal and H. Segur (unpublished).

${ }^{23}$ D. A. Kessler, J. Koplik, and H. Levine, Phys. Rev. A 33, 3352 (1986).

${ }^{24}$ A. Barbieri, D. C. Hong, and J. S. Langer, Phys. Rev. A 35, 1802 (1987).

${ }^{25}$ B. Caroli, C. Caroli, B. Roulet, and J. S. Langer, Phys. Rev. A 33, 442 (1986).

${ }^{26}$ B. Caroli, C. Caroli, C. Misbah, and B. Roulet, J. Phys. (Paris) 47, 1623 (1986), and unpublished.

${ }^{27}$ D. Meiron, Phys. Rev. A 33, 2704 (1986).

${ }^{28}$ D. C. Hong and J. S. Langer, Phys. Rev. A 36, 2325 (1987); pointed bubble solutions were also studied by J. $M$. Vanden-Broeck, Phys. Fluids 29, 1343 (1986).

${ }^{29}$ Y. Couder, O. Cardoso, D. Dupuy, P. Tavernier, and W. Thom, Europhys. Lett. 2, 437 (1986).

${ }^{30}$ Y. Couder, N. Gérard, and M. Rabaud, Phys. Rev. A 34, 5175 (1986).

${ }^{31}$ T. Maxworthy, J. Fluid Mech. 173, 95 (1986).

${ }^{32}$ Note in this respect that although some of the modes given in Eq. (4) can also be obtained from the integro-differential equation for the interface along the lines discussed by $A$. Karma [Phys. Rev. A 34, 4353 (1986)], such an analysis is much more involved than the one discussed here. Apparently, a counting argument is easier to implement for the partial differential equations than for the integro-differential equations.

${ }^{33}$ A. J. DeGregoria and L. W. Schwartz, Phys. Rev. Lett. 58, 1742 (1987).

${ }^{34}$ See Ref. 9 of Segur and Kruskal, Ref. 12.

${ }^{35} \mathrm{H}$. Segur (private communication).

36J. Coron, C. R. Acad. Sci. Paris 294, 127 (1982).

${ }^{37}$ The uniqueness of the solutions in the half-space has also been shown by Weinstein, Ref. 11 .

${ }^{38}$ M. D. Kruskal, in Nonlinear Wave Motion, edited by A. C. Newell (American Mathematical Society, Providence, 1974). 